\title{
XXIII.
}

\section{Zusatz zu dem Referate über die Abhandlung des Herrn Tschirjew.}

Von

\author{
Prof. C. Westphal.
}

\begin{abstract}
Aus
uf die Theorie der Ataxie, welche Herr Dr. Tschirjew im Ansehlusse an die interessanten Resultate seiner physiologischen Versuche aufzustellen versucht hat, habe ich bereits in meiner ersten Arbeit über die Sehnenphänomene hingewiesen. Es heisst daselbst*): „Die Versuchung liegt nahe, die obigen Ausfübrungen für eine Theorie der Ataxie zu verwerthen, wobei der Mangel der mechanischen Erregbarkeit der Muskeln durch Dehnung, auf die Antagonisten einer intendirten Bewegung bezogen, eine Rolle zu spielen hätte. Ich will es jedoch dem Leser überlassen, die Gedanken, die hieran anzuknüpfen wären, sich selbst weiter auszuführen" u. s. w. Der obige Satz, den Herr 'Tschirjew übersehen zu haben scheint, en thält bereits seineTheorie; er sagt nämlich, etwas weitläuftiger ausgedrückt, Folgendes: wenn Muskeln (wie die Abhandlung zeigen sollte) durch Dehnung zur Contraction zu bringen sind und diese Fähigkeit in einer Beziehung zu dem in ihnen vorhandenen Tonus steht, bei der Ataxie aber (in der Tabes), wie in der Abhandlung gezeigt, ihr Tónus und ihre Fähigkeit durch Dehnung sich zu contrahiren verloren geht, so könnte man versucht sein, die Ataxie in der Tabes auf Grund die Annahme zu erklären, dass im physiologischen Zustande bei jeder Bewegung die Antagonisten mechanisch gedehnt werden, durch diese Dehnung in einen gewissen Zustand der Contraction gerathen und dadurch auf die Heftigkeit und das Slossweise der intendirten Bewegung moderirend und hemmend wirken. Sind die Muskeln schlaff, ohne Tonus, wie bei der Tabes, ziehen sie sich demgemäss nicht mehr bei mechanischer Dehnung zusammen, so fällt diese hemmende Wirkung der Antagonisten fort: anstatt einer normalen Beugung des
\end{abstract}

*) Dieses Archiv Bd. V. S. 834. 
Vorderarms z. B. wird dieselbe stossweise und excessiv, weil der Triceps, in Folge seiner Einbusse an Tonus, nicht mehr durch die mechanische Dehnung bei Beugung des Vorderarms in Contraction gerather und dadurch mässigend auf die Beugung einwirken kann.

Dieser in dem angeführten Satze meiner Arbeit enthaltene Gedanke liegt auch der Theorie des Herrn T schirjew von der Ataxie zu Grunde, nur dass letzterer noch auf die Verhinderung der elastischen Schwankungen durch denselben Vorgang Rücksicht genommen hat. Die Frage, woher der Tonus der Muskeln rührt, ist hierbei natürlich gleichgültig.

Ich selbst habe diese Theorie, die sich mir unwillkürlich aufdrängen musste, nicht weiter ansgeführt, weil mir die nächste Ueberlegung zeigte, dass sie vollständig unhaltbar; ich mochte sie nicht erst zum Leben erwecken, um sie sogleich wieder begraben zu müssen. Es mag genügen, Herrn Tschirjew auf die Erfahrungen bei Lähmung peripherischer Nerven und bei partiellen Muskelatrophien zu verweisen. Hier, wo der Tonus gewisser Muskeln doch oft gründlich genug aufgehoben ist, sieht man keine Ataxie der Bewegungen, bei denen die gelähmten oder atrophischen Muskeln als Antagonisten fungiren müssten. Schon durch diese Thatsache allein wird, wie mir scheint, die in Rede 'stehende Theorie der Ataxie hinfällig; indess liesse sich noch eine Reihe anderer Argumente dagegen anführen, die an dieser Stelle zu entwickeln zu weitlänfig wäre.

Bei der herrschenden Neigung, Theorien über pathologische Vorgänge, auf die Physiologie gestützt, ohne gehörige Prüfung zu acceptiren, möchte ich durch vorstehende Zeilen von vorn berein auf das Ungenügende der in Rede stehenden zur Erklärung des pathologischen Phänomens der Ataxie bei der Tabes hingewiesen haben. 